\title{
Analysis of a novel MEMS-based design of micro-direct methanol fuel cell
}

\author{
Falin Chen ${ }^{a}$, Min-Hsing Chang ${ }^{b, *}$, Hung-Yi. Lin ${ }^{a}$ \\ a Institute of Applied Mechanics, National Taiwan University, Taipei 107, Taiwan, ROC \\ ${ }^{\mathrm{b}}$ Department of Mechanical Engineering, Tatung University, 40, 3rd Section, \\ Chun-Shan North Road, Taipei 104, Taiwan, ROC
}

Received 15 October 2007; received in revised form 8 December 2007; accepted 10 December 2007

Available online 23 December 2007

\begin{abstract}
A theoretical analysis is performed in this study for a novel MEMS-based design of micro-direct methanol fuel cell which was proposed by Motokawa et al. [S. Motokawa, M. Mohamedi, T. Momma, J. Electrochem. Commun. 6 (2004) 562-565]. The system comprises two parallel microchannels and is fabricated by a series of steps of MEMS techniques. The methanol stream occupies one channel as fuel and the saturated oxygen liquid stream flows in the other channel as oxidant. Both reactants are dissolved in dilute sulfuric acid solution. The top of the system is covered by a layer of Nafion membrane as the electrolyte layer. Such a novel design eliminates the complicated water management problem and could potentially enhance the volume power density. The cell performance is investigated in detail by examining the effects of several system parameters. The present results provide significant physical insights for the system and benefit the further optimal design of this novel MEMS-based design of microfuel cell for the fulfillment of practice application in portable power sources.
\end{abstract}

(C) 2008 Elsevier B.V. All rights reserved.

Keywords: Micro-direct methanol fuel cell; MEMS-based design; Microchannel

\section{Introduction}

Microfuel cells based on MEMS technology have received much attention recently due to their widely promising applications in portable power sources [1-15]. Particularly, many studies focused on the development of low power direct methanol fuel cells (DMFCs) and provided several different designs [1-11]. However, most researches in the literature were experimental investigations and the system performance was tested by limited operation conditions only. In order to explore the overall system characteristics, it is necessary to perform a theoretical investigation and find the best way to optimize the cell performance. In this study, we develop a theoretical model to simulate a novel design of micro-MFC which was proposed by Motokawa et al. [1]. The system comprises two parallel microchannels and could be fabricated by a series of

\footnotetext{
* Corresponding author. Tel.: +886 $225925252 \times 3410 / 512$; fax: +886225997142 .

E-mail address: mhchang@ttu.edu.tw (M.-H. Chang).
}

steps from MEMS techniques. One channel is for the methanol stream as fuel and the other is for the saturated oxygen liquid stream as oxidant. Both reactants are dissolved in dilute sulfuric acid solution and flow in parallel through the microchannels. The top of the system is covered by a layer of Nafion membrane as the proton exchange layer. Such a novel design possesses many merits: the planar structure allows it possible to integrate the anode and cathode onto the same side of a substrate; both the fuel and oxidant streams are isolated in different microchannels and thus avoid the possible fuel crossing problem; the path for the protons to travel from anode to cathode stream is short which reduces the effect of ohmic losses; the catalyst layer can be coated on the bottom and side-walls of the microchannel which increases the reaction surface. Therefore, it is important to understand the characteristics of this novel microfuel cell system.

Accordingly, we perform a numerical study to investigate the species transport in this system and explore the characteristics of cell performance. The effects of several critical parameters are examined in detail. The results provide an overview of physical insights for the transport phenomena in the system and will 
be helpful for the further improvement of this novel design of micro-DMFC.

\section{Theoretical model and formulation}

The detailed system configuration is shown in Fig. 1(a) and the cross-sectional view including the insulated silicon substrate is indicated in Fig. 1(b). Both methanol and oxygen streams enter the system at two separate inlets and flow in parallel through the microchannels. The system dimensions indicated in Fig. 1(a) are the same as those used in the experimental study [1]. Both methanol and oxygen are dissolved in $0.5 \mathrm{M}$ sulfuric acid solutions. The assumption of natural laminar flow within the microchannel is employed and the catalyst layers in both anode and cathode microchannels are assumed to be coated on the surface of substrate in U-shape as shown in Fig. 1(b). It is assumed that electrochemical reactions occur in these layers only. In the anode side, the methanol will react as the half-reaction below

$\mathrm{CH}_{3} \mathrm{OH}+\mathrm{H}_{2} \mathrm{O} \rightarrow \mathrm{CO}_{2}+6 \mathrm{H}^{+}+6 \mathrm{e}^{-}, \quad E_{0}=0.02 \mathrm{~V}$

(a)

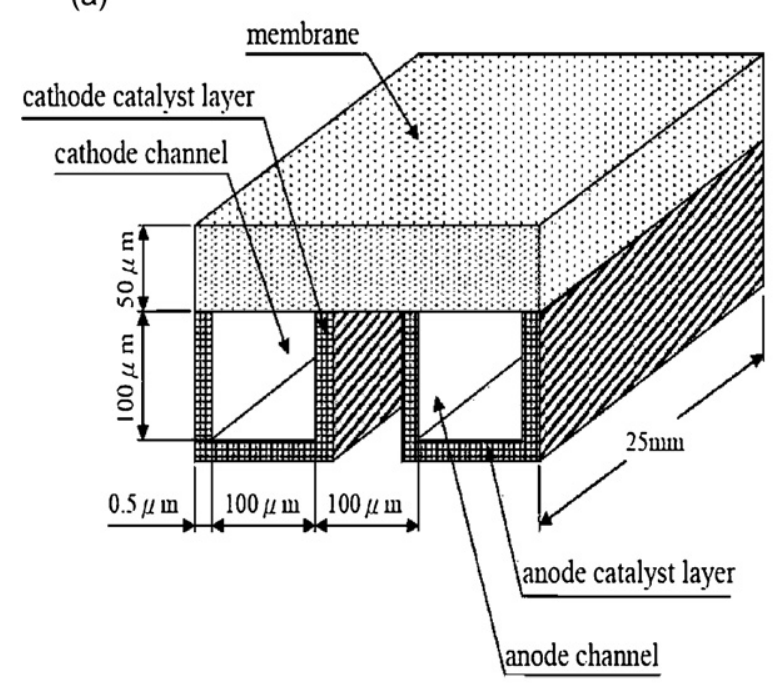

(b)

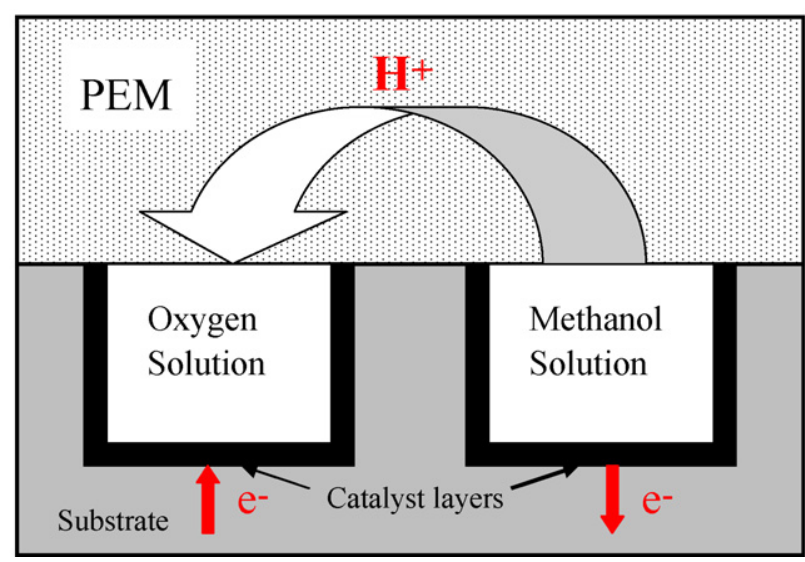

Fig. 1. (a) The diagram of system configuration; (b) the cross-sectional view of system and indication of working principle.
The protons will be transferred to the cathode side through the covered Nafion membrane and the released electrons will reach the cathode via an external circuit. The oxygen dissolved in the cathode stream will react with the protons and electrons and result in the half-reaction in the cathode side

$\frac{3}{2} \mathrm{O}_{2}+6 \mathrm{H}^{+}+6 \mathrm{e}^{-} \rightarrow 3 \mathrm{H}_{2} \mathrm{O}, \quad E_{0}=1.23 \mathrm{~V}$

By combining both half-reactions (1) and (2), this device can generate electricity and the overall electrochemical reaction is the same as the conventional DMFC below

$\mathrm{CH}_{3} \mathrm{OH}+\frac{3}{2} \mathrm{O}_{2} \rightarrow \mathrm{CO}_{2}+2 \mathrm{H}_{2} \mathrm{O}, \quad E_{0}=1.21 \mathrm{~V}$

In order to simplify the simulation model, the cell is assumed to operate under $1 \mathrm{~atm}, 25^{\circ} \mathrm{C}$ and the flows are isothermal and incompressible. Accordingly, the governing phenomena in this micro-DMFC system involve the transports of mass, momentum, reactant, and charge. Within the microchannels, the governing equations of liquid streams can be described by the continuity equation, Navier-Stokes equation, and Fick's law as given below

$\nabla \cdot \mathbf{U}=0$,

$\rho\left[\frac{\partial \mathbf{U}}{\partial t}+\nabla \cdot(\mathbf{U} \mathbf{U})\right]=-\nabla p+\mu \nabla^{2} \mathbf{U}$,

$\rho\left[\frac{\partial}{\partial t} Y_{i}+\nabla \cdot \mathbf{U} Y_{i}\right]=\nabla \cdot \mathbf{J}_{i}$,

where $\rho$ is the fluid density, $\mathbf{U}$ the flow velocity, $p$ the pressure, and $\mu$ the dynamic viscosity, $Y_{i}$ the mass fraction of $i$-th species, and $\mathbf{J}_{i}$ is the diffusion flux. As for the catalyst layers and membrane, the governing equations can be expressed by

$$
\begin{aligned}
\frac{\partial}{\partial t}(\varepsilon \rho)+\nabla \cdot(\varepsilon \rho \mathbf{U})=0, & \\
\frac{\partial}{\partial t}(\varepsilon \rho \mathbf{U})+\nabla \cdot(\varepsilon \rho \mathbf{U} \mathbf{U})= & -\varepsilon \nabla p+\nabla \cdot(\varepsilon \boldsymbol{\tau})-\frac{\varepsilon^{2} \mu \mathbf{U}}{\kappa} \\
& -\frac{\varepsilon^{3} C_{\mathrm{F}} \rho}{\sqrt{\kappa}}|\mathbf{U}| \mathbf{U},
\end{aligned}
$$

$\frac{\partial}{\partial t}\left(\varepsilon \rho Y_{i}\right)+\nabla \cdot\left(\varepsilon \rho \mathbf{U} Y_{i}\right)=\nabla \cdot \mathbf{J}_{i}+\dot{\omega}_{i}$,

where $\varepsilon$ is the porosity, $\boldsymbol{\tau}$ the shear force tensor, $\kappa$ the permeability, $C_{\mathrm{F}}$ the quadratic drag factor, and $\dot{\omega}_{i}$ is the production rate of the $i$-species. The last two terms of Eq. (8) represent the effect of additional drag force imposed by the pore walls on the fluid within the pores, which usually results in a significant pressure drop across the porous structure. It is noted that in the case of pure fluid with $\varepsilon \rightarrow 1$ and $\kappa \rightarrow \infty$, these equations could be reduced to the same forms as Eqs. (4)-(6). The term $\dot{\omega}_{i}$ in Eq. (9) is the source term due to electrochemical reaction in the catalyst layer. It should be zero in the membrane domain. The charge transport is characterized by the electric field and in the liquid streams the electric field can be described by

$\nabla \cdot \mathbf{i}=0$, 
where $\mathbf{i}$ is the vector of current density. This equation indicates the continuity of current within the liquid streams. While in the catalyst layers and membrane, the electric fields can be defined by

$\nabla \cdot \mathbf{i}_{\mathrm{F}}+\nabla \cdot \mathbf{i}_{\mathrm{S}}=0$

where $\mathbf{i}_{\mathrm{F}}$ is the current density in the polymer electrolyte (ionic phase), and is is the current density in the solid or electronic phase of the porous matrix. Since the electrochemical reaction occurs within the catalyst layer only, for a catalyst layer with porous structure the current densities $\mathbf{i}_{\mathrm{F}}$ and $\mathbf{i}_{\mathrm{S}}$ should satisfy the relationship below

$-\nabla \cdot \mathbf{i}_{\mathrm{F}}=\nabla \cdot \mathbf{i}_{\mathrm{S}}=S$,

where $S$ is the reaction rate in terms of unit $\mathrm{Am}^{-3}$. Furthermore, the Bulter-Volmer equation is used to estimate the loss of overvoltage and can be written in the general form

$S_{i}=a j_{0}\left(\frac{C_{i}}{C_{i}^{0}}\right)^{\beta_{i}}\left[\exp \left(\frac{\alpha_{\mathrm{a}} n F}{R T} \eta\right)-\exp \left(-\frac{\alpha_{\mathrm{c}} n F}{R T} \eta\right)\right]$,

where $a$ is the density of the catalyzed active area on the electrode, $j_{0}$ the exchange current density at the reference reactant concentration $C_{i}^{0}, C_{i}$ the concentration of species $i, \beta_{i}$ the reaction order, $\alpha_{\mathrm{a}}$ and $\alpha_{\mathrm{c}}$ are the charge transfer coefficient, respectively for the anode and cathode reactions, $n$ the number of electrons transferred in the reaction, $R$ the universal gas constant, $T$ the absolute temperature, and $\eta$ is the overvoltage. The commercial CFD package, CFD-ACE+, is employed in the present investigation to perform the numerical analysis. The system is discretized with structured grids and then solved by the upwind scheme based on the finite volume method. In order to make sure the numerical accuracy, several sets of grid number have been tested and finally the converged results are determined by an economic consumption of CPU time.

\section{Results and discussion}

In this section we first compare the numerical results with the experimental data [1] and subsequently analyze the effect of each system parameter on the cell performance. The parameters used in both anode and cathode microchannels are given in Table 1 and the properties of membrane are list in Table 2. All the parameters are acquired from the literature [1,16-19] and the same as the operating condition in Ref. [1] except the porosity of catalyst layer which is assumed to be 0.4 . As shown in Fig. 2, the present numerical results are in good agreement with the experimental data [1]. Note that during the simulation process, the flow rate and reactant concentrations at the inlets must be specified first. An assigned cell potential is then given to determine its corresponding current density. This process is repeated several times to obtain the polarization curve. In Table 1 the inlet flow rate, concentration of reactant, and porosity and thickness of catalyst layer are adjustable. Their effects on cell performance will be demonstrated in this section. The results in Fig. 2 also indicate that the cell performance depends on the activity of anode catalyst layer. In the case of anode catalyst
Table 1

Parameters used in anode and cathode microchannels [1,16-18]

\begin{tabular}{lll}
\hline & Anode & Cathode \\
\hline Inlet flow rate $\left(\mu 1 \mathrm{~min}^{-1}\right)$ & 10 & 10 \\
Concentration of methanol $(\mathrm{M})$ & 2 & - \\
Concentration of oxygen $(\mathrm{mM})$ & - & 4 \\
Conductivity of solution $\left(1 \Omega^{-1} \mathrm{~m}^{-1}\right)$ & 20.692 & 20.42 \\
Density of solution $\left(\mathrm{kg} \mathrm{m}^{-3}\right)$ & 1004.909 & 1021.819 \\
Diffusion coefficient $\left(\mathrm{m}^{2} \mathrm{~s}^{-1}\right)$ & $1.28 \times 10^{-9}$ & $2.42 \times 10^{-9}$ \\
Porosity of catalyst layer & 0.4 & 0.4 \\
Thickness of catalyst layer $(\mu \mathrm{m})$ & 0.5 & 0.5 \\
Tortuosity of catalyst layer & 1.5 & 1.5 \\
Charge transfer coefficient & 0.15 & 0.25 \\
$\quad$ Exchange current density $\left(\mathrm{A} \mathrm{m}{ }^{-2}\right)$ & $5.41 \times 10^{-4}$ & $5 \times 10^{-4}$ \\
$\quad$ for Pt catalyst & & \\
Exchange current density $\left(\mathrm{A} \mathrm{m}^{-2}\right)$ & $10^{-3}$ & - \\
$\quad$ for Pt-Ru catalyst & & \\
\hline
\end{tabular}

Table 2

Parameters of proton exchange membrane $[1,19]$

\begin{tabular}{ll}
\hline Conductivity of ion $\left(1 \Omega^{-1} \mathrm{~m}^{-1}\right)$ & 8.3 \\
Permeability of membrane $\left(\mathrm{m}^{2}\right)$ & $1.8 \times 10^{-18}$ \\
Porosity & 0.28 \\
Tortuosity & 7 \\
\hline
\end{tabular}

layer composed of elements $\mathrm{Pt}$ and $\mathrm{Ru}$, the surface exchange current density is higher than the case of containing Pt only and thus results in better cell performance. In the following discussions, the operating condition of Table 1 with the Pt-Ru anode catalyst layer is taken as the base state to explore the effect of each parameter.

The effects of reactant concentrations are first examined and the variations of concentrations down the microchannels are demonstrated in Fig. 3(a) and (b), respectively, for methanol and oxygen at a typical condition with cell potential $230 \mathrm{mV}$. Obviously, the variation of methanol concentration is insignificant down the stream under steady state as indicated in Fig. 3(a). We also observe that the methanol indeed diffuses gradually into

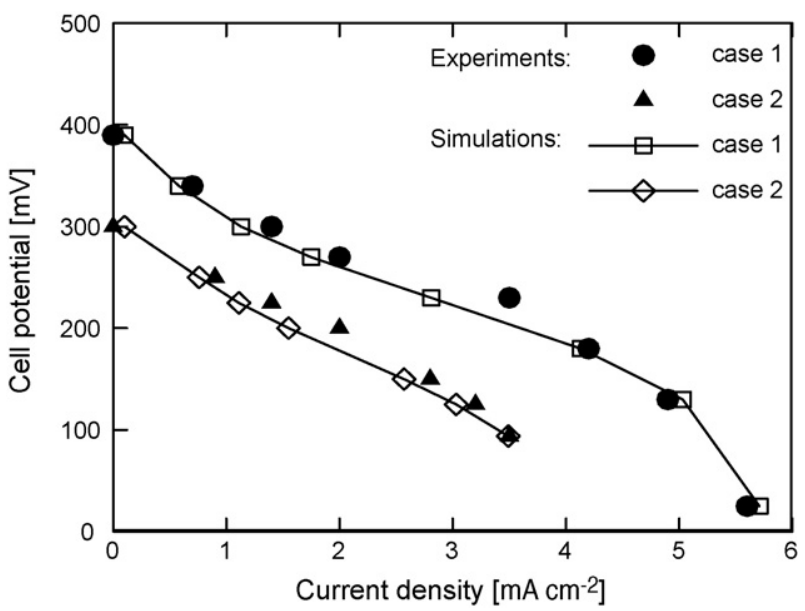

Fig. 2. Comparisons of present results with the experimental data of Motokawa et al. [1], where case 1 is the Pt-Ru anode catalyst layer and case 2 is the $\mathrm{Pt}$ anode catalyst layer. 

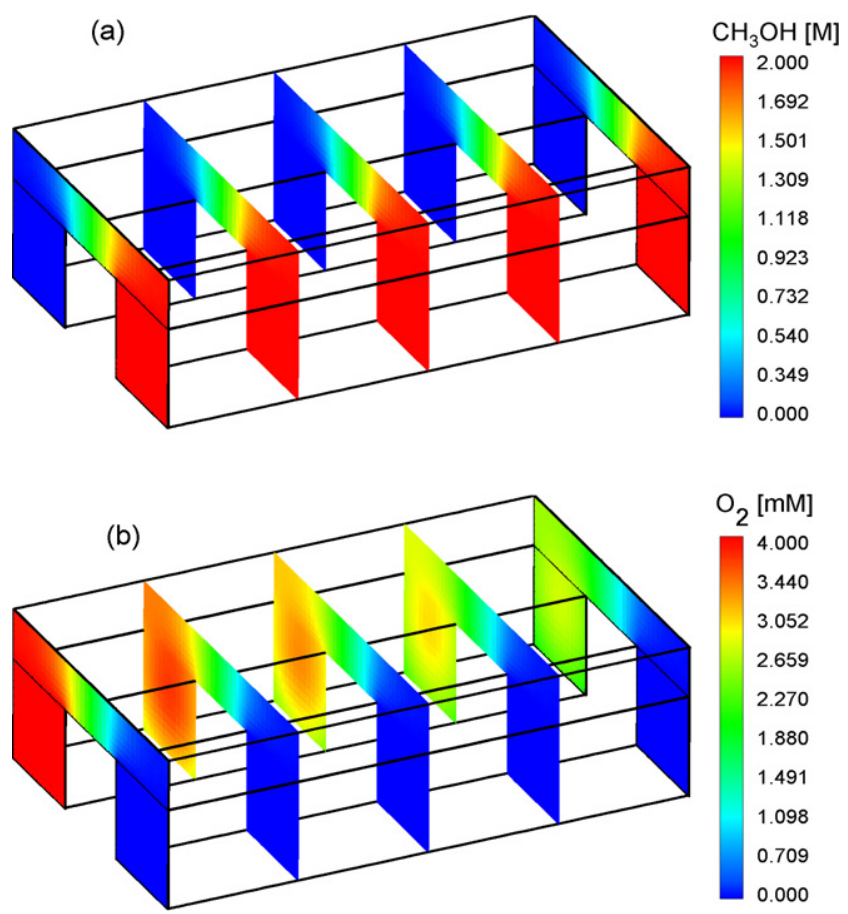

Fig. 3. The concentration variations of reactants down the stream at cell potential $230 \mathrm{mV}$; (a) the methanol concentration; (b) the oxygen concentration.

the covering membrane layer. However, it never enters the cathode stream and due to the U-shaped coating of catalyst layer as shown in Fig. 1(a), the fuel crossover problem which occurs in conventional DMFC could be ignored in this novel design. In contrast, the oxygen concentration varies significantly down the stream as shown in Fig. 3(b). The concentration boundary layer adjacent to the catalyst layer develops significantly down the flow direction. It will increase the resistance of mass transfer of reactant to the reaction site especially in the region near the outlet of microchannel. This result implies that the mass transfer loss of cell potential would be primarily dominated by the cathode stream. Therefore, how to reduce the mass transfer loss in cathode stream would be a critical issue for this design of microfuel cell.

The effects of methanol and oxygen concentrations on the cell performance are shown in Fig. 4(a) and (b), respectively. In Fig. 4(a), one can see that the cell performance is almost independent of the variation of methanol concentration, while it depends heavily on the variation of oxygen concentration as indicated in Fig. 4(b). These results reveal that the cell performance can be improved greatly by using a higher concentration of oxygen solution. The limiting current density is almost in proportion to the oxygen concentration. However, it is generally difficult to raise the oxygen concentration in a liquid solution unless the system can operate under high pressure conditions. Thus, in order to reach the objective that the cell performance of this design would be comparable to an air-breathing DMFC, it is quite important to find an efficient way for the raise of oxygen concentration in cathode stream.

The volumetric flow rate is also a vital factor in the operation of microfuel cell. Its influence on the cell performance is shown
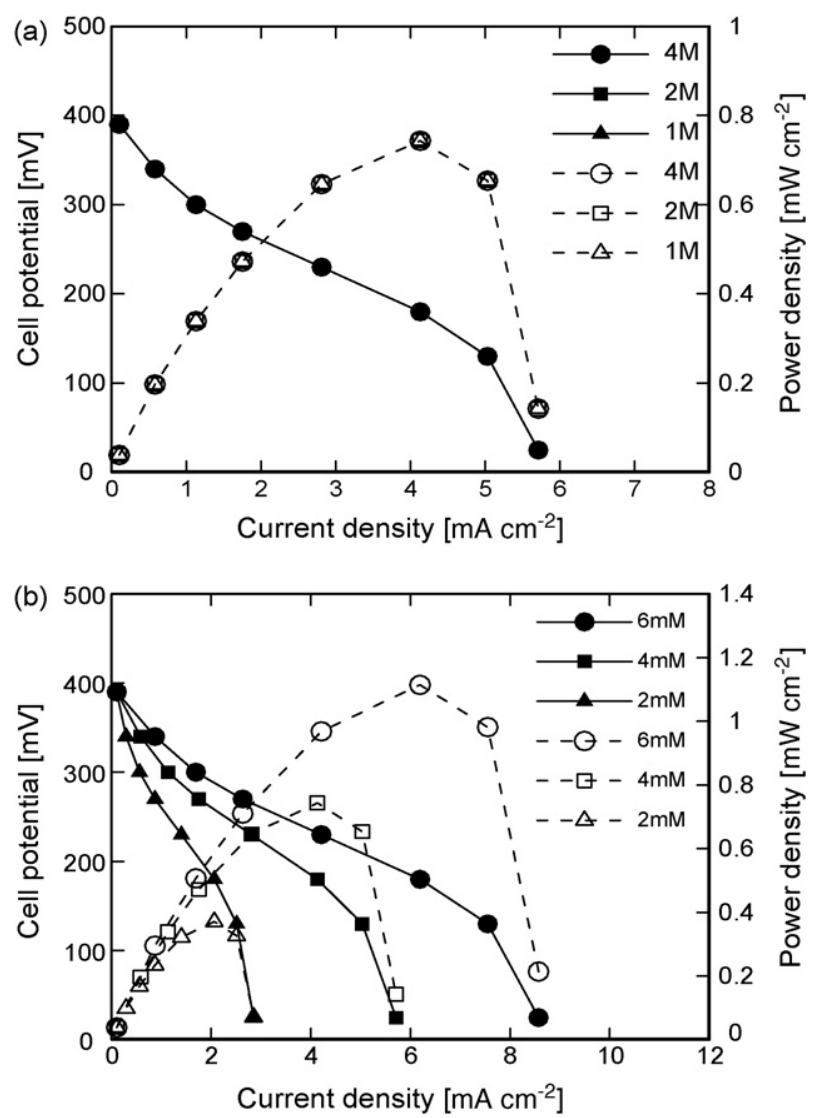

Fig. 4. The effect of reactant concentration on the cell performance; (a) three typical cases of methanol concentration; (b) three typical cases of oxygen concentration.

in Fig. 5. Obviously, the cell performance can be enhanced by operating the microfuel cell with a higher volumetric flow rate. The main reason can be explained by observing the variations of oxygen concentration down the stream for two typical volumetric flow rates as illustrated in Fig. 6(a) and (b). When the cell is operated under a higher flow rate, the liquid stream passes the microchannel more quickly and the growth of concentration boundary layer down the stream is relatively slower, which results in a thinner concentration boundary layer at the outlet and

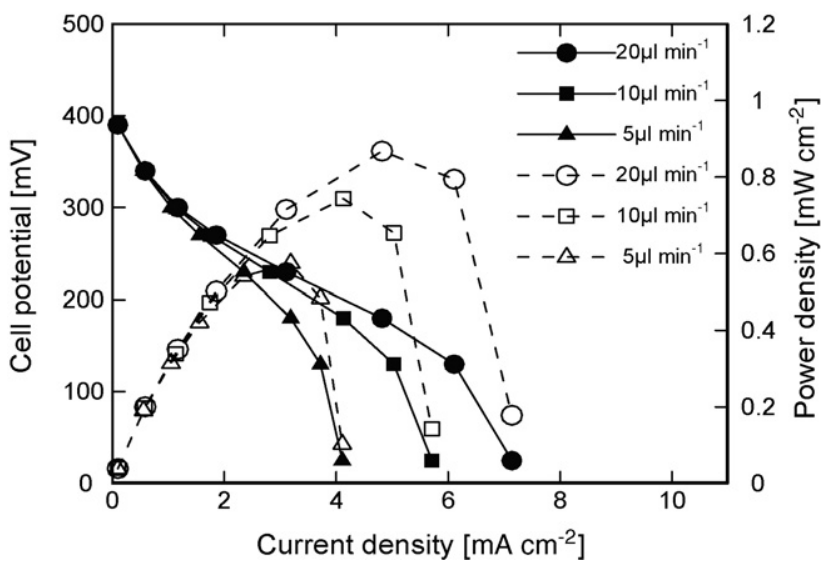

Fig. 5. The variations of $I-V$ curve and power density for three assigned volumetric flow rates. 

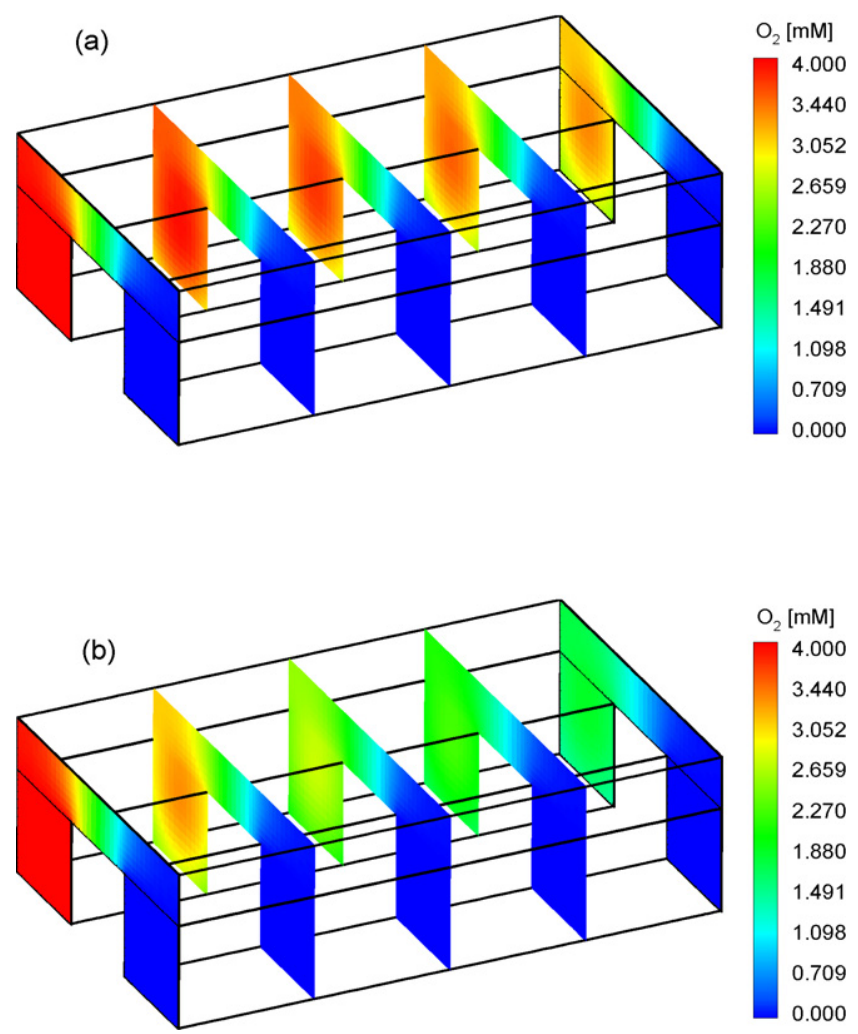

Fig. 6. The variations of oxygen concentration down the stream for two typical volumetric flow rates at cell potential $230 \mathrm{mV}$; (a) $20 \mu 1 \mathrm{~min}^{-1}$; (b) $5 \mu 1 \mathrm{~min}^{-1}$.

a higher oxygen concentration in the central flow. Accordingly, the transport resistance of oxygen to the catalyst layer will be reduced and more oxygen can be transferred to the region near the outlet. The concentration losses will decrease significantly and therefore the cell performance will be improved. While in the case of lower flow rate in Fig. 6(b), it takes more time for the flow passing through the microchannel and the liquid stream will have more time to be in contact with the catalyst layer. Apparently, the concentration boundary layer grows more rapidly down the stream which makes a quite low oxygen concentration in the rear part of microchannel. This result will cause severe concentration losses as we have discussed in Fig. 3(b). As a result, we may conclude that a higher volumetric rate is necessary for the raise of cell performance. However, this effect is limited when the volumetric flow rate is over a certain critical value. As shown in Fig. 7, for a given oxygen concentration in cathode stream, it is found that the limiting current density will increase quickly at first with the volumetric flow rate but approach a constant gradually once the flow rate is large enough. Similar phenomena can be observed in all three typical cases of Fig. 7. This result reveals that the effect to compensate the concentration losses in the rear section of microchannel decays gradually with the volumetric flow rate and an over high flow rate is not necessary and beneficial for the cell performance.

The porosity and thickness are both important characteristics of the catalyst layer especially in the cathode side. Their influences on the cell performance are demonstrated in Figs. 8 and 9, respectively. A higher porosity may reduce the transport resis-

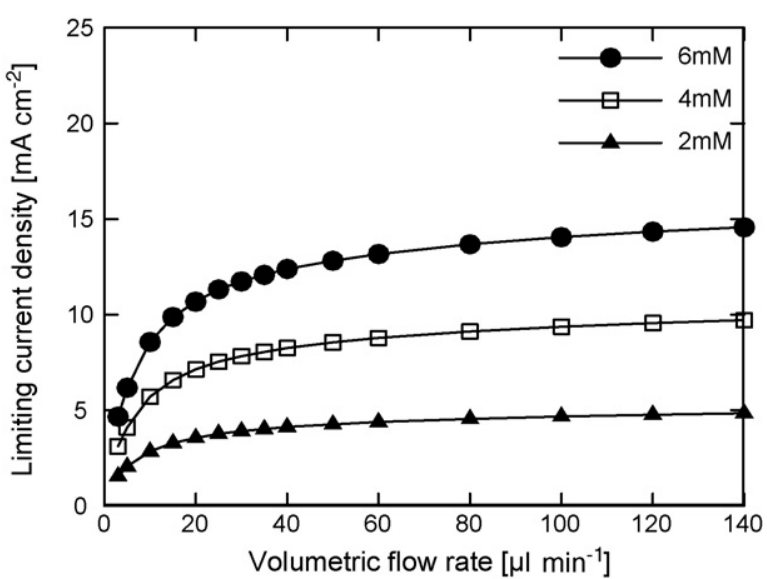

Fig. 7. The variations of limiting current density with volumetric flow rate for three assigned oxygen concentrations.

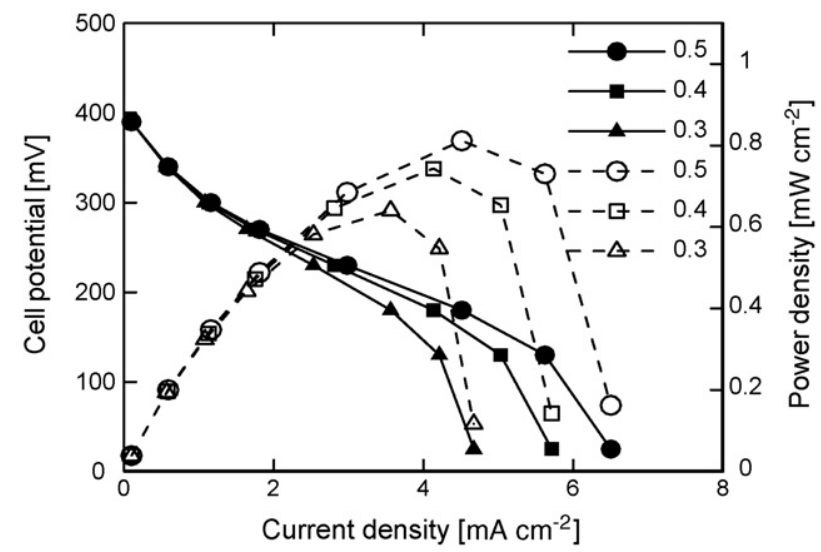

Fig. 8. The variations of cell performance for three assigned porosities of cathode catalyst layer.

tance of oxygen because the reactant can penetrate into the catalyst layer more easily to react with the catalytic particles. Thus, the catalyst layer with higher porosity will appear to have better cell performance as shown in Fig. 8. Similarly, a thicker cathode catalyst layer will provide more reacting sites for the electrochemical reaction and thus the density of catalyzed active area will increase on the electrode surface. It is clear from

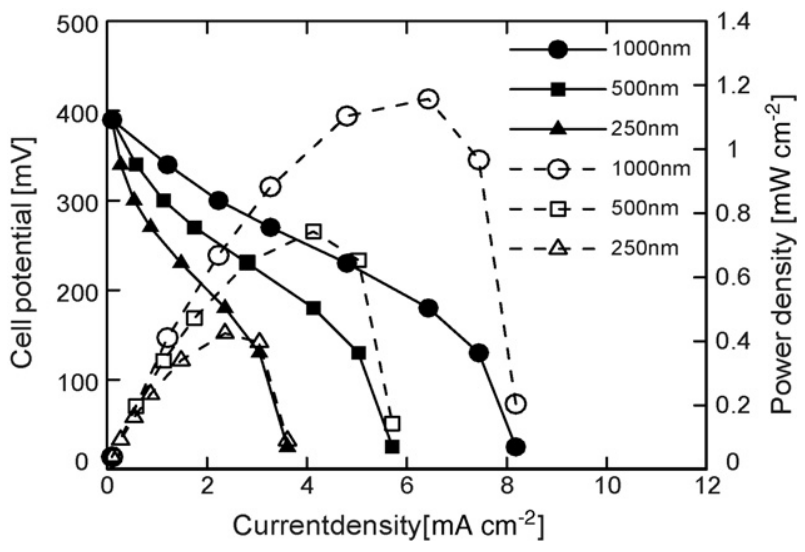

Fig. 9. The variations of cell performance for three assigned thicknesses of cathode catalyst layer. 
the Bulter-Volmer equation that the cell potential losses will decrease with the same current density under this condition. So a thicker cathode catalyst layer will effectively increase the cell performance as illustrated in Fig. 9.

The oxygen consumption rate $\lambda$ is also an important parameter to evaluate the cell performance. It can be defined as

$\lambda=\frac{\dot{m}_{i}-\dot{m}_{\mathrm{e}}}{\dot{m}_{i}} \times 100 \%$,

where $\dot{m}_{i}$ and $\dot{m}_{\mathrm{e}}$ are respectively the mass flow rate of oxygen at the entrance and outlet of the microchannel. It is found that the variations of $\lambda$ are insignificant in all the adjustments of system parameters discussed above except the thickness of cathode catalyst layer. The results are shown in Fig. 10 for three typical thicknesses. As seen in this figure, a thicker cathode catalyst layer will also enhance the oxygen consumption rate with the same volumetric flow rate. Note that the value of $\lambda$ will decrease first and then tend to be a constant with increasing of flow rate. Although the cell has high oxygen consumption rate under low flow rate conditions, the cell performance is still poor due to the serious concentration losses as we have discussed in Fig. 6(b). In comparison with Fig. 7, it is obvious that when the volumetric flow rate exceeds the critical value, both limiting current density and oxygen consumption rate will become independent of the volumetric flow rate.

The improvement of geometric design is also a feasible method to enhance the cell performance. One possible way is to lengthen the length of microchannel. Since the overall reaction area increases, the total power output will also increase. However, it is predictable that under the same flow rate condition, the mass transfer losses will be more pronounced for a longer microchannel and thus the average current density will decrease as well as the cell performance. The other possible way is to adjust the aspect ratio of the cross-sectional view of the microchannel. Here we consider three typical aspect ratios and the results for cell performance and oxygen concentration at the outlet of microchannel are shown in Figs. 11 and 12, respectively. The aspect ratio is defined as the ratio of channel height over channel width. Note that the cross-sectional area

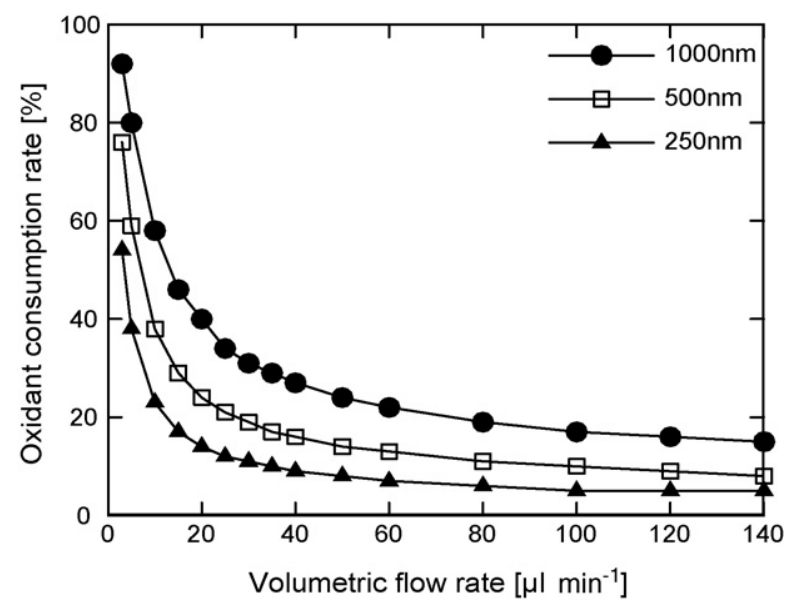

Fig. 10. The variations of oxygen consumption rate with volumetric flow rate for three typical thicknesses of cathode catalyst layer.

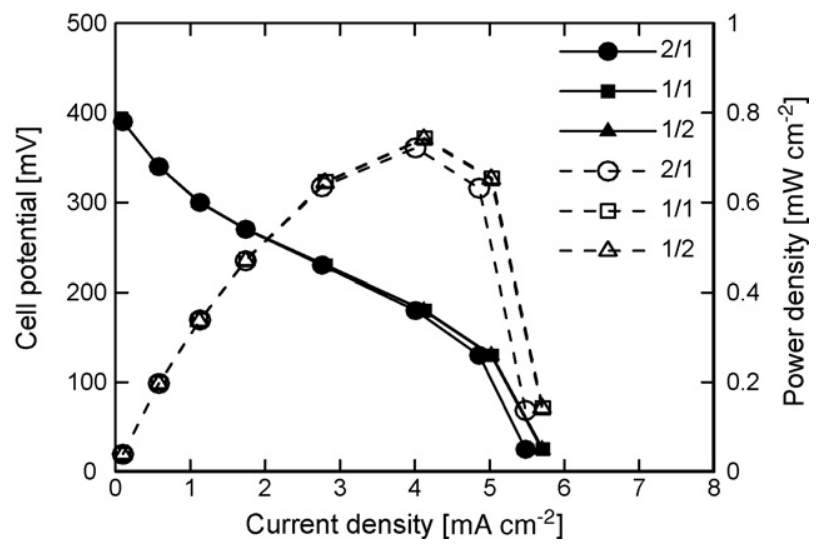

Fig. 11. The variations of cell performance for three assigned aspect ratios of microchannel.

(a)2/1

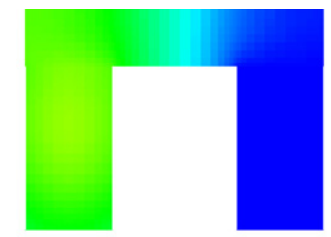

(b) $1 / 1$

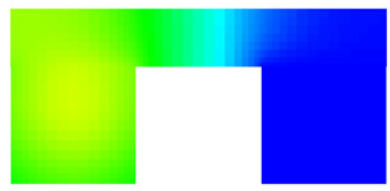

(c) $1 / 2$

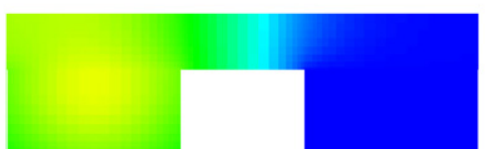

Fig. 12. The profiles of oxygen concentration at the outlet of microchannel for three typical aspect ratios with the same volumetric flow rate.

and the volumetric flow rate are fixed in these cases. One can see that the concentration loss is slightly higher in case (a) as indicated in Fig. 12, and the case (c) with lower aspect ratio seems to possess better cell performance as shown in Fig. 11. But the changes indeed are quite small which indicate the cell performance is not quite sensible to the variation of aspect ratio.

\section{Conclusions}

We have implemented a theoretical analysis for the novel design of micro-DMFC proposed by Ref. [1]. The results show that the cell performance is mainly restricted by the cathode stream in which the oxygen concentration is relatively too low to provide sufficient power output. A higher volumetric flow rate is found to be able to reduce the transport resistance of oxygen as well as the induced concentration losses. However, this effect is limited and there exists an optimal volumetric flow rate that an extra increase of flow rate over this optimal value is not necessary. Raising the oxygen concentration in the cathode stream is the most efficient way to enhance the cell performance. But it is generally difficult to dissolve more oxygen in a liquid 
solution. A possible treatment is to operate the system under high pressure condition. However, it will result in a potential difficulty in practical applications for portable power systems. The properties of catalyst layer are also the dominant factors for the cell performance. A catalyst layer with high porosity will benefit the improvement of cell performance. Besides, an increase of thickness of catalyst layer will provide more reaction sites on the electrode surface per unit area and thus the cell performance could be improved. As for the geometric design, it is found that the aspect ratio of microchannel does not play an important role in the factors affecting the cell performance. Further studies focusing on how to raise the cell performance as high as an air-breathing DMFC will be the most critical issue in the future.

\section{Acknowledgements}

The financial support for this research from National Science Council of Taiwan through the grants NSC 96-2212-E-036-041 and NSC 96-2628-E-002-073-MY3 are gratefully acknowledged.

\section{References}

[1] S. Motokawa, M. Mohamedi, T. Momma, J. Electrochem. Commun. 6 (2004) 562-565.
[2] Y. Zhang, J. Lu, S. Shimano, H. Zhou, R. Maeda, J. Electrochem. Commun. 9 (2007) $1365-1368$.

[3] S.K. Kamarudin, W.R.W. Daud, S.L. Ho, U.A. Hasran, J. Power Sources 163 (2007) 743-754.

[4] J. Yeom, R.S. Jayashree, C. Rastogi, M.A. Shannon, P.J.A. Kenis, J. Power Sources 160 (2006) 1058-1064.

[5] X. Liu, C. Suo, Y. Zhang, X. Wang, C. Sun, L. Li, L. Zhang, J. Micromech. Microeng. 16 (2006) S226-S232.

[6] Y. Jiang, X. Wang, L. Zhong, L. Liu, J. Micromech. Microeng. 16 (2006) S233-S239.

[7] K.B. Min, S. Tanaka, M. Esashi, J. Micromech. Microeng. 16 (2006) 505-511.

[8] S.C. Yao, X.D. Tang, C.C. Hsieh, Y. Alyousef, M. Vladimer, G.K. Fedder, C.H. Amon, Energy 31 (2006) 636-649.

[9] S. Motokawa, M. Mohamedi, T. Momma, S. Shoji, T. Osaka, Electrochemistry 73 (2005) 346-351.

[10] H.Y. Cha, H.G. Choi, J.D. Nam, Y. Lee, S.M. Cho, E.S. Lee, J.K. Lee, C.H. Chung, Electrochim. Acta 50 (2004) 795-799.

[11] G.Q. Lu, C.Y. Wang, X. Zhang, Electrochim. Acta 49 (2004) 821-828

[12] J.D. Morse, Int. J. Energy Res. 31 (2007) 576-602.

[13] B.Y. Park, M.J. Madou, J. Power Sources 162 (2006) 369-379.

[14] Y. Yamazaki, Electrochim. Acta 50 (2004) 663-666.

[15] K.B. Min, S. Tanaka, M. Esashi, Electrochemistry 70 (2002) 924-927.

[16] D.R. Lide, CRC Handbook of Chemistry and Physics, CRC Press, 1975.

[17] G.B. Jung, A. Su, C.H. Tu, Y.T. Lin, F.B. Weng, S.H. Chan, J. Power Sources 171 (2007) 212-217.

[18] W. Vielstich, H.A. Gasteiger, A. Lamm, Handbook of Fuel Cells-Fundamentals Technology and Applications, John Wiley \& Sons, 2003.

[19] F.B. Weng, A. Su, G.B. Jung, J. Power Sources 145 (2005) 546-554. 